\section{Ejercicio adaptado: el ejercicio físico como} herramienta terapéutica

\section{Adapted exercise: physical exercise as a therapeutic tool}

\section{Sr. Editor:}

Uno de los principales objetivos sanitarios de la Organización Mundial de la Salud -OMS- es la reducción de la incidencia y mortalidad de las Enfermedades Crónicas No Transmisibles -ENT-, particularmente en los países de economías emergentes, donde se cuenta el $80 \%$ de los muertos anualmente por estas enfermedades a nivel mundial ${ }^{1}$. A pesar de que los indicadores macroeconómicos y sanitarios de Chile lo sitúan en condición similar a los países desarrollados, la realidad para la mayoría de las personas con ENT es la realidad de la atención primaria, donde la mayor parte de la intervención médica se centra en la farmacoterapia, con un rol menor de las intervenciones no farmacológicas, particularmente de la prescripción de ejercicio, a pesar de ser la medida más costo-efectiva para la prevención primaria y secundaria en estas enfermedades ${ }^{2}$.

La necesidad de intervención física en esta población requiere una aproximación altamente especializa$\mathrm{da}$, desde el conocimiento de la fisiopatología a la teoría y metodología del entrenamiento, que permita aplicar el "stress controlado" del ejercicio físico a un sistema fisiológico con bajas reservas funcionales y, en general, frágil $^{3}$. Pese a lo anterior, los programas de pregrado de medicina en las universidades chilenas no consideran la formación académica en ésta área y sólo hace algunos años existe dentro del programa de las escuelas de Kinesiología y Terapia en Actividad Física una dedicación desde la formación de pregrado, forzando al sistema a contar con profesionales con postgrado para intentar cubrir esta necesidad, tanto de las áreas citadas, como Profesores de Educación Física que se han especializado en intervenciones en salud. Considerando que los médicos atienden al 100\% de estos pacientes, la mayor parte de la indicación de actividad física para control de las ENT se realiza con un mínimo de conocimiento, probablemente por una formación orientada a la terapéutica y no a la prevención.

El uso del ejercicio como terapia es tan antiguo como la medicina misma, pero el concepto de "ejercicio como fármaco" empieza a utilizarse desde la década del 1980 como analogía a la forma de prescribir ejercicio en relación a los medicamentos, tanto como en los efectos positivos para la salud. Este concepto es especialmente útil para los médicos, pues mejora la comprensión de los componentes del ejercicio y permite indicaciones precisas a los pacientes, siendo utilizado para su enseñanza en cursos internacionales como el Exercise is Medicine, esfuerzo conjunto del Colegio Americano de Medicina del Deporte-ACSM- y la Asociación Médica Americana 
-AMA-. Lamentablemente, el uso de esta analogía, que hoy se utiliza en algunos centros para la promoción de sus programas de actividad física, implica poner en riesgo el objeto preventivo de estas intervenciones por equiparar al ejercicio a un estándar de tratamiento.

En contraste a lo anterior, plantear el ejercicio y su relación con la salud desde las habilidades físicas, permite intervenir transversalmente al usuario, con una acción tanto preventiva como terapéutica, donde se cambia el foco desde el resultado clínico al resultado físico, entendiendo que la modificación de las habilidades físicas implica un impacto directo y proporcional en el estado de salud. En este sentido, aplicar el ejercicio como fármaco no es inconveniente, siempre que su uso no esté restringido para el tratamiento de una condición de salud en específico.

Las recomendaciones internacionales proponen la necesidad continua de entrenamiento físico, pero en una realidad social donde sólo una de cada nueve personas hace ejercicio en forma regular ${ }^{4}$, trabajar las cualidades físicas implica retrasar o evitar la aparición de ENT y la disfuncionalidad que acompaña a la senescencia, con un alto impacto sanitario potencial. A diferencia de los sujetos sanos, un sujeto que inicia un programa de entrenamiento con pérdida funcional establecida, requiere un trabajo adaptado a la situación específica de su capacidad física, intervención que no puede limitarse al diagnóstico específico o la habilidad física perdida. Es ejemplo recurrente la búsqueda de mejoras en el consumo pico de oxígeno -VO2peak- en un paciente con obesidad o diabetes mellitus tipo 2, falla asociada al déficit del metabolismo energético de los hidratos de carbono y lípidos. Si nos dedicamos exclusivamente a trabajar con ejercicios aeróbicos como caminata, baile o bicicleta recreativos, abandonamos parte sustantiva de la mejora potencial que se puede alcanzar en términos de calidad de vida en este sujeto, por ejemplo, por la falta de trabajo de fuerza que también impacta positivamente en el resultado metabólico ${ }^{5,6}$.

Trabajar en esta población con ejercicio adaptado permite mejorar las cualidades físicas a través de respuestas adaptativas al entrenamiento. Implica adecuada prescripción, control y progresión del tipo y carga de ejercicio y con esto, lograr mejoras en el estado de salud, la capacidad y reserva funcional, la corrección de alteraciones metabólicas, neurológicas y locomotoras. Se diferencia del uso del ejercicio como fármaco en el inherente cambio del foco sobre la patología para centrarse en la persona, con capacidades físicas y estado de salud deteriorado, pregonando el trabajo integral de estas habilidades en todos los sujetos, haciendo ahínco en aquellas más deficitarias y en la carga de antecedentes sanitarios, sin perder de vista la fisiopatología. El uso de este concepto también implica redefinir la condición del sujeto; cuando alguien que se consideraba previamente enfermo y restringido de actividad física, puede integrarse a un contexto socialmente vinculado a sujetos sanos, sacando la etiqueta de paciente y permitiendo a la persona cambiar la percepción de su estado de salud, con una capacidad funcional reducida, pero en proceso de recuperación.

Entender la condición de salud desde el paradigma de las cualidades físicas permite enfrentar a cualquier usuario y lograr mejoras funcionales significativas con impacto medible en calidad de vida y parámetros sanitarios analíticos, con o sin diagnósticos establecidos, con un mínimo de efectos adversos e impactando positivamente en la carga sanitaria de la población intervenida, en forma paralela y complementaria a otras intervenciones en el estilo de vida, el estudio completo del paciente y la farmacoterapia requerida.

\section{Arnaldo Burdiles-Alvarez $z^{1,3}$, Alexis Espinoza-Salinas ${ }^{2,3, a}$ ${ }^{1}$ Centro de Medicina del Deporte, Clínica Las Condes. \\ ${ }^{2}$ Laboratorio de Fisiología del Ejercicio, Escuela de Kinesiología, Universidad Santo Tomás, Santiago, Chile. \\ ${ }^{3}$ Centro de Ejercicio Adaptado, Asociación Cristiana de Jóvenes - YMCA, Santiago. ${ }^{a}$ Kinesiólogo, Profesor de Educación Física, MSc.}

\section{Referencias}

1. Organización Mundial de la Salud. Informe Mundial Sobre la Diabetes. Ginebra. 2016; (4)1-4.

2. The Diabetes Prevention Program Research Group.Reduction in the incidence of type 2 diabetes with lifestyle intervention or metformin.N Engl J Med. 2002;346(6):393-403

3. López J \& López L. Fisiología clínica del ejercicio. Buenos aires, Argentina: Editorial médica Panamericana; 2008: 35-43.

4. Ministerio de Salud, Chile. Segunda Encuesta Nacional de Salud. 2009-2010.

5. Pesta D. Goncalves R. Madiraju A. Strasser B. Sparks L. Resistance training to improve type 2 diabetes: working toward a prescription for the future. Nutrition \& Metabolism. 2017; 14: 24

6. Shiroma E, Cook N, Manson J, Moorthy M, Buring J, Rimn E, Lee I. Strength Training and the Risk of Type 2 Diabetes and Cardiovascular Disease. Med. Sci. Sports Exerc 2017; 49(1): 40-6.

Arnaldo Burdiles Alvarez arnaldo@burdiles.cl

Teléfono: +569-98218319

Código postal:

Centro de Medicina del Deporte - CLC, Estoril \#450, Ed. Rojo Piso

4, Las Condes. 